\title{
Parafuso intramedular de redução fechada em fraturas das falanges proximais dos dedos da mão: Uma série de três casos*
}

\section{Closed-Reduction Intramedullary Screw in Fractures of the Proximal Phalanges of the Digits of the Hand: A Series of Three Cases}

\author{
Arsanto Triwidodo $^{10}$ Hendar Nugrahadi Priambodo ${ }^{10}$ Yogi Ismail Gani ${ }^{10}$ \\ ${ }^{1}$ Departamento de Ortopedia e Traumatologia, Faculdade de \\ Medicina, Universitas Indonesia, Koja Public Regional Hospital, \\ Jacarta, Indonésia \\ Rev Bras Ortop \\ Endereço para correspondência Yogi Ismail Gani, Department of \\ Orthopaedic and Traumatology, Faculty of Medicine Universitas \\ Indonesia, Dr. Cipto Mangunkusumo Hospital, Jl. Diponegoro no. 71, \\ Jakarta, 10430, Indonésia \\ (e-mail: yogiismailgani@yahoo.com).
}

\section{Resumo \\ Palavras-chave \\ - falanges dos dedos da mão \\ - fraturas ósseas \\ - placas ósseas \\ - parafusos ósseos \\ - fios ortopédicos \\ - fixação de fratura interna}

As lesões esqueléticas mais frequentes são as fraturas que ocorrem nas mãos, que representam aproximadamente $20 \%$ de todas as fraturas. As mais comuns são as fraturas falangeanas, que abrangem $6 \%$ de todas as fraturas. As fraturas da falange proximal aparecem com mais frequência. O principal objetivo do atendimento é corrigir a anatomia, reduzir os danos aos tecidos moles, e mobilizar os dedos atingidos assim que a estabilização da fratura permitir. Este relato tem como objetivo examinar os efeitos clínicos e da radiação nas fraturas da falange proximal, em pacientes infectados com a fixação de parafuso intramedular que foram submetidos a redução fechada. Este é o relato de três casos consecutivos de fratura da falange: duas fraturas da base e uma fratura simples da diáfise. Todos foram submetidos a redução fechada com parafuso intramedular de compressão sem cabeça. A magnitude pré-operatória da angulação da falange proximal foi em média $30,3^{\circ}$ (variação de $13^{\circ}$ a $42^{\circ}$ ). Dois pacientes apresentaram deformidade rotacional. Os achados clínicos foram medidos com a versão abreviada do questionário de Disfunções do Braço, Ombro e Mão (Quick Disabilities of the Arm, Shoulder and Hand, QuickDash, em inglês), em que foram avaliados a amplitude de movimento e os resultados funcionais. Complicações foram

* Trabalho desenvolvido no Departamento de Ortopedia e Traumatologia, Faculdade de Medicina, Universitas Indonesia, Jacarta, Indonésia.

recebido

30 de Dezembro de 2020 aceito

11 de Fevereiro de 2021
DOI https://doi.org/ 10.1055/s-0041-1739405. ISSN 0102-3616. (c) 2021. Sociedade Brasileira de Ortopedia e Traumatologia. All rights reserved.

This is an open access article published by Thieme under the terms of the Creative Commons Attribution-NonDerivative-NonCommercial-License, permitting copying and reproduction so long as the original work is given appropriate credit. Contents may not be used for commercial purposes, or adapted, remixed, transformed or built upon. (https://creativecommons.org/ licenses/by-nc-nd/4.0/)

Thieme Revinter Publicações Ltda., Rua do Matoso 170, Rio de Janeiro, RJ, CEP 20270-135, Brazil 
observadas em um período mínimo de 3 meses. Os pacientes apresentavam flexoextensão ativa total na articulação interfalangiana proximal e na articulação metacarpofalangiana de flexo-extensão sem deformidade rotacional. A pontuação no QuickDASH foi satisfatória (média: 2,3; variação: 0 a 4,5). Não houve cirurgias secundárias, e não se observaram complicações. A fixação intramedular das fraturas da falange proximal com o parafuso de tensão canulado tem se mostrado uma cirurgia segura, com excelente desempenho funcional e recuperação precoce. A fratura é minimizada e consolidada adequadamente pelos pacientes.

\section{Abstract}

\section{Keywords}

- finger phalanges

- fractures, bone

- bone plates

- bone screws

- bone wires

- fracture fixation internal
The most frequent skeletal injuries are hand fractures, which constitute around $20 \%$ of all fractures. Fractures of the phalanx are usual, comprising $6 \%$ of all fractures. Proximal phalanx fractures arise more often. The main goals of the care are to repair the anatomy, reduce the damage to soft tissue, and mobilize the damaged fingers as soon as stabilization of the fracture allows it. The present report is intended to examine the clinical and radiation effects of proximal phalanx fractures in patients treated with intramedullary screw fixation who underwent closed reduction. We report three consecutive cases of proximal phalanx fracture: two basal fractures and one simple shaft fracture. They were treated surgically with closed reduction with intramedullary headless compression screws. The preoperative magnitude of the angulation of the proximal phalanx averaged $30.3^{\circ}$ (range: $13^{\circ}$ to $42^{\circ}$ ). Two patients presented rotational deformity. The clinical findings were measured using the abbreviated version of the Disabilities of the Arm, Shoulder and Hand (Quick-DASH) questionnaire, and the range of motion and functional results were assessed. Complications were observed over a span of at least 3 months. The patients displayed complete active flexion-extension proximal interphalangeal joint and flexion-extension metacarpophalangeal joint without rotative deformity. The scores on the QuickDASH were satisfactory (mean: 2.3; range: 0 to 4.5). No secondary surgeries were performed, and complications were not observed. Intramedullary fixation of proximal phalanx fractures with cannulated tension screws has been shown to be a safe operation, resulting in outstanding functional performance and an early recovery. The fracture is minimized and properly consolidated by the patients.

\section{Introdução}

As fraturas das mãos são reconhecidamente as lesões esqueléticas mais frequentes, e são responsáveis por 20\% de todas as fraturas. ${ }^{1,2}$ As fraturas falangeanas representam $6 \%$ de todas as fraturas. Os objetivos primários do tratamento são: corrigir a anatomia, evitar lesões desnecessárias dos tecidos moles, e permitir a mobilização precoce do sítio da lesão. ${ }^{3}$

A solução geral para as fraturas falangeanas é a seguinte: usar a técnica de solidarização ou tala, fraturas extra-articulares saudáveis, tratamento cirúrgico das partes articulares deslocadas, desalinhamento rotacional, ou encurtamento $>15^{\circ}$ ou $6 \mathrm{~mm} .{ }^{4}$ Os procedimentos cirúrgicos podem incluir redução fechada e pinagem ou redução aberta e fixação. A redução fechada e a fixação interna fornecem compressão interfragmentária e uma alternativa de mobilização mais precoce, ao passo que a redução aberta e a fixação externa fornecem apenas compressão interfragmentária. ${ }^{5}$

Relatamos três casos de fraturas da falange proximal da mão submetidas ao método de redução fechada com a fixação de parafuso intramedular e à mobilização precoce.

\section{Métodos}

Relatamos três casos de fratura da falange proximal: duas fraturas transversais da base da falange, e uma fratura transversal simples da diáfise. Todos os pacientes eram destros, e um deles apresentava lesão na mão não dominante. Foi realizada uma avaliação radiográfica da mão (-Fig. 1). Todas as fraturas foram fechadas, e não houve associação com lesão neurovascular. Todos os pacientes foram submetidos a redução fechada com a fixação de parafuso intramedular.

\section{Técnica Cirúrgica}

As cirurgias foram realizadas por um ortopedista sênior em um único hospital universitário, usando uma técnica idêntica em todas elas. $\mathrm{O}$ diâmetro do canal intramedular de cada paciente foi avaliado por meio de radiografia na incidência de perfil. Usamos um parafuso de 2,2 mm de diâmetro em cada cirurgia. Foram utilizadas as técnicas anterógrada e a intraarticular em todos os dedos. 

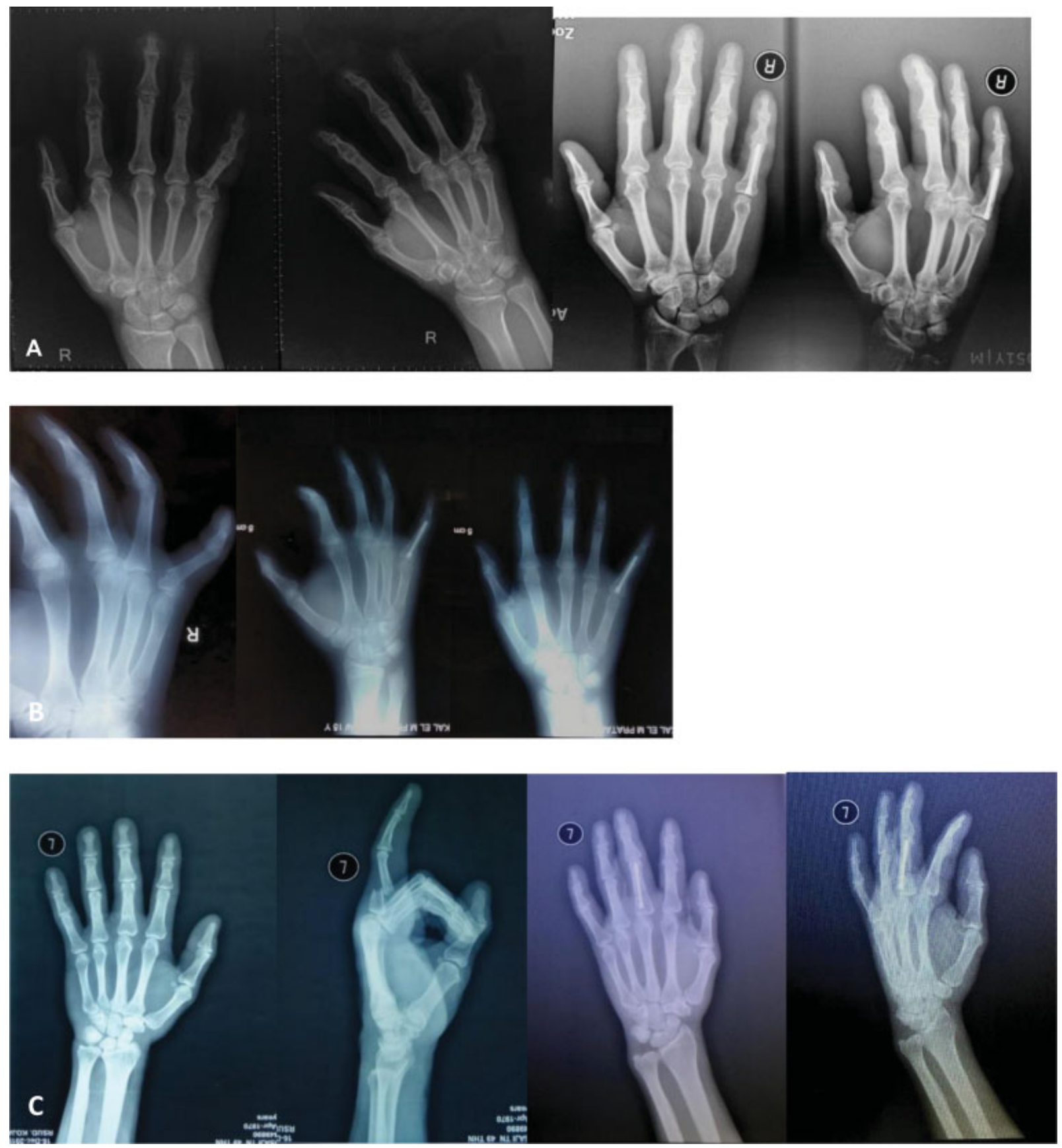

Fig. 1 Avaliações radiológicas pré-operatória (esquerda) e pós-operatória (direita): (A) Caso 1. (B) Caso 2. (C) Caso 3.

A redução das fraturas foi realizada sob fluoroscopia, sendo corrigida por tração e manipulação do dedo em caso de deformidade rotacional. Foi realizada uma incisão de $2 \mathrm{~cm}$, seguida de uma capsulotomia dorsal, a fim de expor a metacarpofalangeana (MCF) intra-articular, conforme mostrado na - Figura 2. Um fio guia foi inserido na porção dorsal central da base da falange proximal, com a articulação MCF com aproximadamente $60^{\circ}$ de flexão. A base do dedo foi suavemente movida dorsalmente para que o fio pudesse ser colocado.

Uma vez que o fio guia estava no devido lugar e tendo sido alcançada uma redução satisfatória, um parafuso canulado sem cabeça foi inserido na metáfise da falange proximal. $O$ fio guia foi removido. $\mathrm{O}$ comprimento do parafuso foi medido por meio de radiografia, e testado mediante fluoroscopia antes da inserção. Tomou-se cuidado especial para garantir que a base do parafuso não se projetasse, e para que a extremidade superior do parafuso atingisse a extremidade do canal medular. A amplitude de movimento (ADM) completa das articulações MCF e interfalangeana proximal (IFP) foi testada antes do fechamento da pele. No pós-operatório, prescreveu-se um regime fisioterapêutico da mão em todos os pacientes.

\section{Avaliação}

Todos os pacientes foram avaliados com um acompanhamento mínimo de 3 meses. No último acompanhamento, foi 

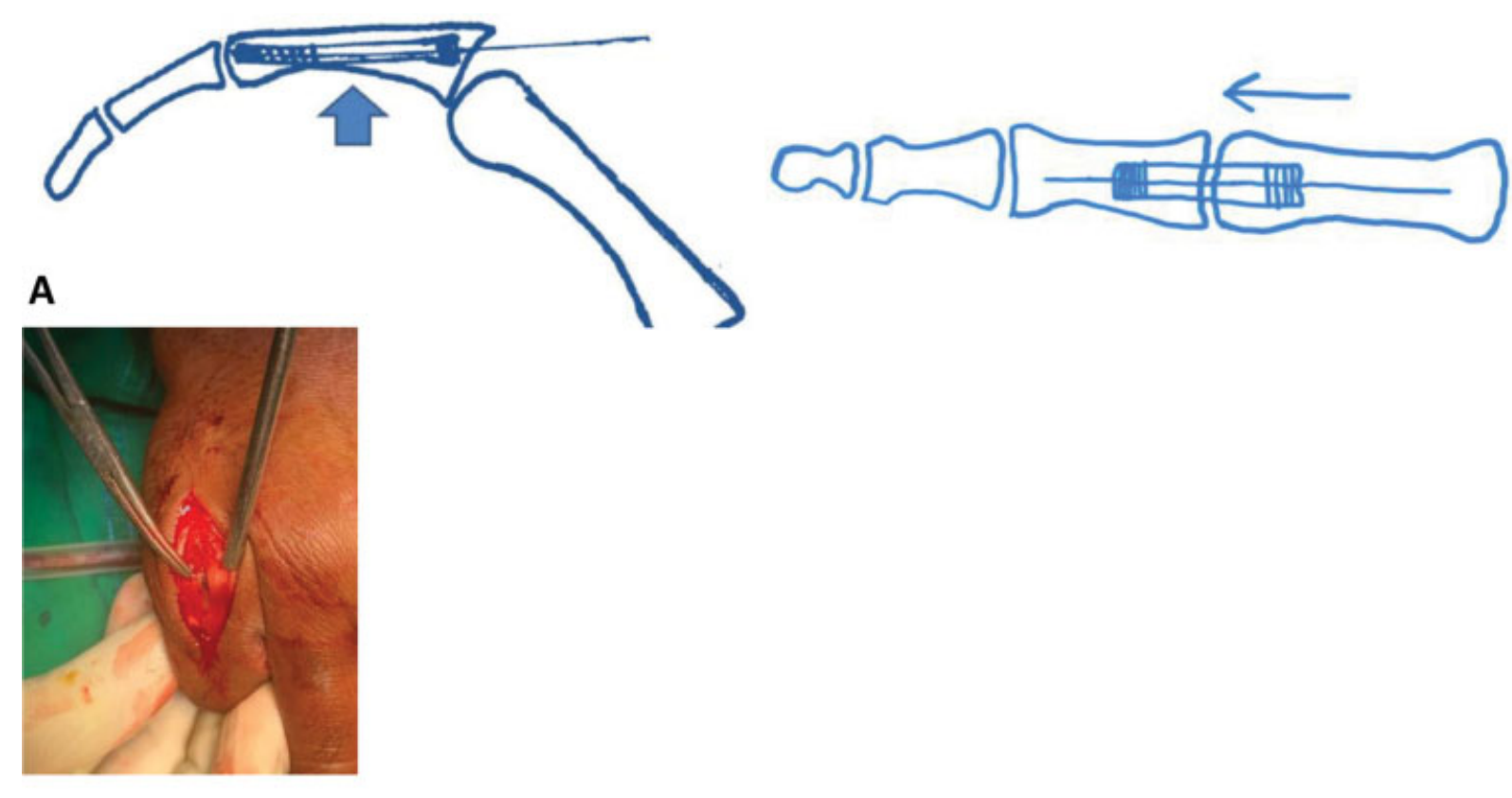

B

Fig. 2 (A) Esquema da técnica intra-articular anterógrada; incidências sagital e axial (B) da incisão do lado dorsal da articulação metacarpofalangeana.

realizada uma avaliação com com a versão abreviada do questionário de Disfunções do Braço, Ombro e Mão (Quick Disabilities of the Arm, Shoulder and Hand, QuickDash, em inglês), em que foram observados quaisquer sintomas persistentes. Foi realizado um exame clínico para avaliar qualquer deformidade, e a ADM ativa das articulações MCF e IFP foi medida com um goniômetro.

\section{Resultados}

Todos os pacientes alcançaram completa ADM ativa e ativoassistida das articulações MCF e IFP na primeira semana do pós-operatório, e demonstraram uma ADM de flexo-extensão ativa total e indolor dessas articulaçõe sem qualquer deformidade (latência prologada/má rotação) no último acompanhamento. A flexão média da articulação MCF foi de $85^{\circ}$ (variação: $80^{\circ}$ a $90^{\circ}$ ), e a flexão média da articulação IFP, $99^{\circ}$ (variação: $90^{\circ}$ a $110^{\circ}$ ). A pontuação média no QuickDASH foi de 2,3 (variação: 0 a 4,5). Não houve queixas quanto à sensibilidade nas articulações MCF e IFP, no sítio da fratura, ou casos de refratura. Nenhum dos pacientes apresentou complicações que exigissem tratamento adicional ou cirurgia secundária. O quadro clínico e os resultados estão resumidos na - Tabela $\mathbf{1}$.

\section{Discussão}

Buscar técnicas menos invasivas tem como objetivo a redução dos riscos, permitindo assim a mobilização precoce do dedo. ${ }^{2}$ A maioria das fraturas falangeanas podem ser tratadas de forma não cirúrgica. No entanto, nos casos aqui descritos, as fraturas eram relativamente instáveis, e necessitavam de cirurgia com parafuso intramedular para a obtenção de um resultado excelente. ${ }^{3,4}$

Em casos de fratura instável do dedo para a qual a cirurgia não é adequada, a operação ideal é incerta. A fixação de placas ajuda a oferecer estabilização para a recuperação precoce, embora os resultados clínicos sejam inconsistentes. ${ }^{5}$ Técnicas minimamente invasivas podem oferecer alternativas viáveis para a fixação das fraturas falangeanas nos tecidos moles. Pode ser usada uma fixação interna ou externa. ${ }^{6}$ Ambas as soluções têm suas próprias armadilhas, associadas à retirada não planejada de hardware, à imobilização, e à fixação incompleta. ${ }^{7}$

O emprego de um método menos invasivo de tratamento das fraturas extra-articulares disfuncionais da falange proximal é mais bem-sucedido e seguro. Análises mostram resultados promissores após a fixação do parafuso intramedular para metacarpos e falanges, com um número reduzido de complicações. ${ }^{8-10}$ Após três meses de acompanhamento, não houve fraturas, deformidades rotacionais, nenhuma doença, condição de dor regional dinâmica, e nenhuma migração de qualquer parafuso. A ADM do dedo foi perfeita. Os resultados revelaram que $75 \%$ da $A D M$ do punho foram recuperados em 2 semanas, sendo que a ADM completa foi alcançada no acompanhamento final.

\section{Conclusão}

Com a fixação de parafuso de compressão intramedular nas fraturas da falange proximal, os pacientes demonstraram um excelente desempenho funcional, e tiveram uma recuperação precoce e completa. As fraturas foram suficientemente reduzidas e consolidadas. As complicações podem ser 
Parafuso intramedular de redução fechada em fraturas das falanges proximais Triwidodo et al.

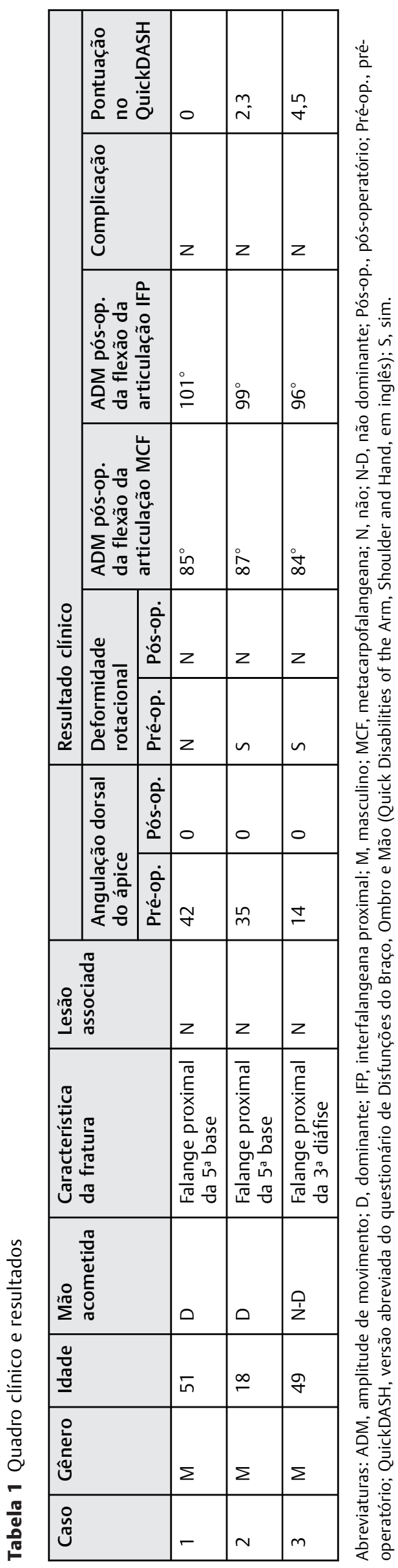

difíceis de tratar, e a não consolidação pode acarretar prejuízos para o resultado funcional.

\section{Suporte Financeiro}

Não houve suporte financeiro de fontes públicas, comerciais, ou sem fins lucrativos.

\section{Conflito de Interesses}

Os autores declaram não haver conflito de interesses.

\section{Referências}

1 Laugharne E, Bhavsar D, Rajaratnam V. The distribution of hand fractures: A British perspective. Eur J Plast Surg 2013;36:367-370

2 Ozer K, Gillani S, Williams A, Peterson SL, Morgan S. Comparison of intramedullary nailing versus plate-screw fixation of extraarticular metacarpal fractures. J Hand Surg Am 2008;33(10): 1724-1731

3 Patankar H, Meman FW. Multiple intramedullary nailing of proximal phalangeal fractures of hand. Indian J Orthop 2008;42 (03):342-346

4 del Piñal F, Moraleda E, Rúas JS, de Piero GH, Cerezal L. Minimally invasive fixation of fractures of the phalanges and metacarpals with intramedullary cannulated headless compression screws. J Hand Surg Am 2015;40(04):692-700

5 Margić K External fixation of closed metacarpal and phalangeal fractures of digits. A prospective study of one hundred consecutive patients. J Hand Surg Br 2006;31(01):30-40

6 Yan YM, Zhang WP, Liao Y, et al. [Analysis and prevention of the complications after treatment of metacarpal and phalangeal fractures with internal fixation]. Zhongguo Gu Shang 2011;24 (03):199-201

7 Orbay JL, Touhami A. The treatment of unstable metacarpal and phalangeal shaft fractures with flexible nonlocking and locking intramedullary nails. Hand Clin 2006;22(03):279-286

8 Giesen T, Gazzola R, Poggetti A, Giovanoli P, Calcagni M. Intramedullary headless screw fixation for fractures of the proximal and middle phalanges in the digits of the hand: a review of 31 consecutive fractures. J Hand Surg Eur Vol 2016;41(07):688-694

9 Held M, Jordaan P, Laubscher M, Singer M, Solomons M. Conservative treatment of fractures of the proximal phalanx: an option even for unstable fracture patterns. Hand Surg 2013;18(02): 229-234

10 Kawamura K, Chung KC. Fixation choices for closed simple unstable oblique phalangeal and metacarpal fractures. Hand Clin 2006;22(03):287-295 